\title{
ADESÃO AO TRATAMENTO DA TUBERCULOSE NO AMAPÁ: UM QUINQUÊNIO DE ANÁLISE EPIDEMIOLÓGICA
}

\section{ARTIGO ORIGINAL}

SANTANA, Jorge Lucas Alves ${ }^{1}$

MAGRINI, Amanda Thais ${ }^{2}$

RIBEIRO, Lucas Almeida ${ }^{3}$

RIGELLI, Roberto Heck ${ }^{4}$

ARAÚJO, Maria Helena Mendonça de ${ }^{5}$

SANTANA, Jorge Lucas Alves. Et al. Adesão ao tratamento da tuberculose no Amapá: Um quinquênio de análise epidemiológica. Revista Científica Multidisciplinar Núcleo do Conhecimento. Ano 05, Ed. 10, Vol. 17, pp. 69-87. Outubro de 2020. ISSN: 2448-0959, Link de acesso: https://www.nucleodoconhecimento.com.br/saude/tratamento-datuberculose

\section{RESUMO}

A tuberculose é uma doença causada pela infecção do Mycobacterium tuberculosis de disseminação por meio de inalação de gotículas de aerossóis, acometendo em

\footnotetext{
${ }_{1}^{1}$ Acadêmico de medicina pela Universidade Federal do Amapá (UNIFAP).

${ }^{2}$ Acadêmico de medicina pela Universidade Federal do Amapá (UNIFAP).

${ }^{3}$ Acadêmico de medicina pela Universidade Federal do Amapá (UNIFAP).

${ }^{4}$ Acadêmico de medicina pela Universidade Federal do Amapá (UNIFAP).

${ }^{5}$ Orientadora. Doutoranda do Programa Acadêmico em Ciências do Cuidado em Saúde - PACCS da Universidade Federal Fluminense (UFF). Professora do Curso de Medicina da Universidade Federal do Amapá (UNIFAP).
} 
grande parte dos casos os pulmões, chamada nesses casos de tuberculose pulmonar, por isso todo paciente que apresenta sintomas respiratórios acima de três semanas, como tosse seca ou produtiva, é considerado suspeito, devendo iniciar a investigação da doença. Essa infecção é um problema de saúde pública de importância principal em países subdesenvolvidos ou em desenvolvimento, ligada a questões sociais e de higiene sanitária. O coeficiente de mortalidade é aproximadamente 2,25 por 100 mil habitantes no Brasil, o que resulta em cerca de 4500 óbitos por ano. O presente estudo tem por objetivo traçar o perfil epidemiológico quantitativamente de forma retrospectiva analisando a adesão ao tratamento por pacientes infectados pelo bacilo de Koch, no estado do Amapá, no recorte temporal de 2015 a 2019, utilizando dados do departamento de informática do Sistema Único de Saúde brasileiro (DATASUS) e correlacionar com informações atuais da literatura. Por meio disso, foram encontradas 1401 notificações de tuberculose no estado, durante esse quinquênio estudado, sendo estas mais recorrentes no sexo masculino e na faixa etária adulta. A taxa de abondo do tratamento mostrou-se similar entre homens e mulheres, com menores índices na faixa etária idosa. Dessa forma, a análise dos dados fornecidos pelo ministério da saúde sobre a temática nesta população mostra-se como uma forma de contribuir com a disseminação de informação sobre a situação epidemiológica amapaense da doença em questão, bem como, atentar os gestores de saúde acerca de medidas de prevenção e manejo mais eficazes no estado.

Palavras-chave: Adesão terapêutica, perfil epidemiológico, tuberculose.

\section{INTRODUÇÃO}

A tuberculose (TB) é uma doença infectocontagiosa provocada pela inalação de aerossóis contendo bacilos de Koch - Mycobacterium tuberculosis - oriundos das vias aéreas de pessoas com doença ativa (pulmonar ou laríngea), chamados bacilíferos, a qual acomete em maior grau, mas não unicamente, os pulmões (KOZAKEVICH e SILVA, 2015).

Relatos históricos indicam que a TB foi introduzida no Brasil por colonizadores, com aumento substancial de sua disseminação durante o período do tráfico negreiro, em 
virtude das péssimas condições médico-sanitárias em que os africanos sequestrados eram transportados. Desde então os índices de mortalidade por TB são extremamente altos (FOCACCIA, 2015).

Durante o século XIX e início do século XX, a tuberculose foi responsável por cerca de $50 \%$ da totalidade de óbitos registrados no Brasil. Em 2019, houve 73.864 novos casos de TB. O coeficiente de mortalidade variou nos últimos dez anos entre 2,2 a 2,3 por 100 mil habitantes, ou seja, o número de óbitos ficou entre 4400 e 4600 por ano. Em 2018, nove estados brasileiros tiveram o coeficiente de mortalidade próximo ou superior ao do país e oito apresentaram coeficientes abaixo de 1,5, dentre eles o Amapá. A TB é considerada pela Organização Mundial da Saúde (OMS) um problema de saúde pública mundial e ela classifica o Brasil em $20^{\circ}$ na lista de países prioritários para a doença (BRASIL, 2020; CARVALHO, 2018; DA SILVA et al., 2018).

Pessoas infectadas pela micobactéria, cuja sintomatologia não é manifestada possuem quadro denominado infecção latente por tuberculose (ILTB), quando os bacilos permanecem inativos no organismo. Existem fatores que podem comprometer o sistema imunológico, aumentando a suscetibilidade do adoecimento (BRASIL, 2018; NOGUEIRA, 2017).

Para realizar o diagnóstico devem-se considerar os grupos de risco (pessoas mais propensas a adquirir determinada patologia), sendo diabéticos, portadores do vírus da imunodeficiência humana (HIV), tabagistas e usuários de álcool ou outras drogas. Diante disso, tais pessoas que apresentem sintomas respiratórios progressivos ou com regressão lenta e radiografia de tórax sugestiva de tuberculose, devem ser avaliadas para a realização da baciloscopia ou do teste rápido molecular (TRM-TB). A baciloscopia é realizada através da análise de duas amostras do escarro do paciente, sendo elas em dois dias consecutivos, enquanto que o TRM-TB é feito por meio de apenas uma amostra de escarro, cultura e teste de sensibilidade. Quando suspeitado ou diagnosticado é necessário notificar (BRASIL, 2017)

As notificações dos casos de TB são importantes para que haja avaliação de controle epidemiológico em nível local, sendo classificadas como agravo de notificação 
compulsória. Existem livros de registros com tais notificações, mas o principal formulário é a ficha de notificação e investigação de tuberculose do Sistema de Informação de Agravos de Notificação (BRASIL, 2018). Quando a tuberculose é citada como principal motivo de óbito ou está associada à morte, deve haver uma comunicação à vigilância epidemiológica do município com o propósito de legitimar esse diagnóstico e averiguar se as ações de vigilância foram realizadas corretamente. No Brasil, 487 casos de TB foram notificados após a morte em 2019, prejudicando a eficiência do Sistema de Vigilância, a garantia do tratamento precoce e a quebra da cadeia de transmissão (BRASIL, 2018; BRASIL, 2020).

A terapêutica medicamentosa está disponível no Sistema Único de Saúde (SUS) e se baseia na associação de fármacos, sendo administrado por dois meses o esquema RIPE (Rifampicina, Isoniazida, Pirazinamida e Etambutol) e durante quatro meses o esquema RH (Rifampcina e Isoniazida), devendo-se ser cumprido rigorosamente para que não ocorra resistência bacteriana (BRASIL, 2019; DA SILVA et al., 2018).

Existem inúmeras adversidades durante o tratamento, como intolerância aos medicamentos, consumo excessivo de álcool, resistência das cepas bacterianas e abandono do uso das medicações. Com isso, foi estabelecido que o tratamento deve ser realizado em regime de Tratamento Diretamente Observado (TDO), sendo necessário que um profissional da saúde supervisione a tomada dos medicamentos diariamente, durante todo o período do tratamento, garantindo uma maior adesão (BRASIL, 2019; KOZAKEVICH e SILVA, 2015).

Após quinze dias do uso da medicação, a chance de transmissão diminui, contribuindo para o aumento de pessoas - uma em cada 10 que iniciam o tratamento - que abandonam o uso das medicações por conta própria. É importante que a terapia medicamentosa não seja interrompida sem a orientação médica, evitando recidiva ou que ocorram complicações que possam levar a óbito (BRASIL 2019). 


\section{METODOLOGIA}

O estudo é do tipo epidemiológico descritivo, transversal e com caráter quantitativo. Para a coleta dos dados, foi utilizado o Departamento de informática do SUS (DATASUS). Por meio deste, foi possível acessar o Sistema de Informação de Agravos e Notificação (SINAN), acerca dos dados sobre diagnósticos da Tuberculose, disponíveis em Informações em Saúde (TABNET), em 14 de julho de 2020.

Os dados sobre os casos de tuberculose diagnosticados foram adquiridos seguindo esta sequencia: No site do DATASUS, clicou-se em "Acesso à informação" e em seguida escolheu-se a opção TABNET; Epidemiológicas e morbidades; Casos de Tuberculose - Desde 2001 (SINAN); Tuberculose desde 2001; na área de abrangência geográfica foi selecionado o Estado do Amapá. Para formar os gráficos foi designado o período de 2015 a 2019. Nos gráficos em que a linha foi selecionada com "Sexo", no DATASUS, as colunas tiveram as seguintes variáveis: "Ano Diagnóstico"; "Confirmação Laboratorial”; "1ª Bac Escarro"; "2a Bac. Escarro"; "Bascilosc $2^{\circ}$ Mês"; "Bascilosc $6^{\circ}$ Mês" e "Situação Encerra.". Já naqueles em que a linha escolhida foi "Situação Encerra.", as variáveis escolhida foram "Ano Diagnóstico" e "Fx Etária".

Para melhor apresentação dos dados, foram feitas algumas reorganizações. $\mathrm{Na}$ variante "Situação Encerrada", foi útil para montar os gráficos somente os tópicos: "Ign/Branco"; "Cura"; "Abandono"; "Óbitos por tuberculose" e "Óbitos por outras causas". Os demais tópicos da variante ("Transferência"; "TB-DR"; "Mudança de esquema"; "Falência" e "Abandono primário") foram compilado em "Outros". $\mathrm{Na}$ variante "Fx Etária" os números foram compilados de acordo com a divisão proposta pelo Instituto Brasileiro de Geografia e Estatística: "Jovens", de 0 a 19 anos; "Adultos", de 20 a 59 anos e "Idosos", para maiores de 60 anos.

A bibliografia foi pesquisada por meio de artigos científicos presentes na biblioteca Scientific Electronic Library Online (SciELO), na base de dados Literatura LatinoAmericana em Ciências da Saúde (Lilacs) e em cartilhas do Ministério da Saúde. As tabelas foram editadas por meio do programa Excel software Microsoft Office 2010. 
Esse estudo não foi submetido ao Comitê de Ética em Pesquisa (CEP), visto que os dados expostos são secundários, isentando o enfoque em pessoas ou instituições, o que atende aos requisitos da Resolução 466 de 2012.

\section{RESULTADOS}

No período analisado - 2015 a 2019 - foram notificados 1401 casos de tuberculose no estado do Amapá, com números crescentes, à exceção de 2018. No diagnóstico, percebe-se uma quantidade expressiva de 411 registros cujo diagnóstico laboratorial não foi realizado. A maior parte das notificações foi no sexo masculino, sendo 898 notificações. Nesse mesmo grupo, percebem-se menores taxas de abandono do tratamento e desfecho de óbito, apesar de também apresentar índices mais baixos de cura, quando comparado ao sexo feminino. Em relação à faixa etária, os adultos (20 a 59 anos) foram o grupo atingido em maior número pela doença, totalizando 1044 casos confirmados, embora os idosos (60 anos ou mais) tenham apresentado maior porcentagem de óbito. Os gráficos a seguir detalham essas variáveis.

Gráfico 1 - Quantidade de casos confirmados por ano de diagnóstico de acordo com o sexo entre, 2015 e 2019 no Amapá.

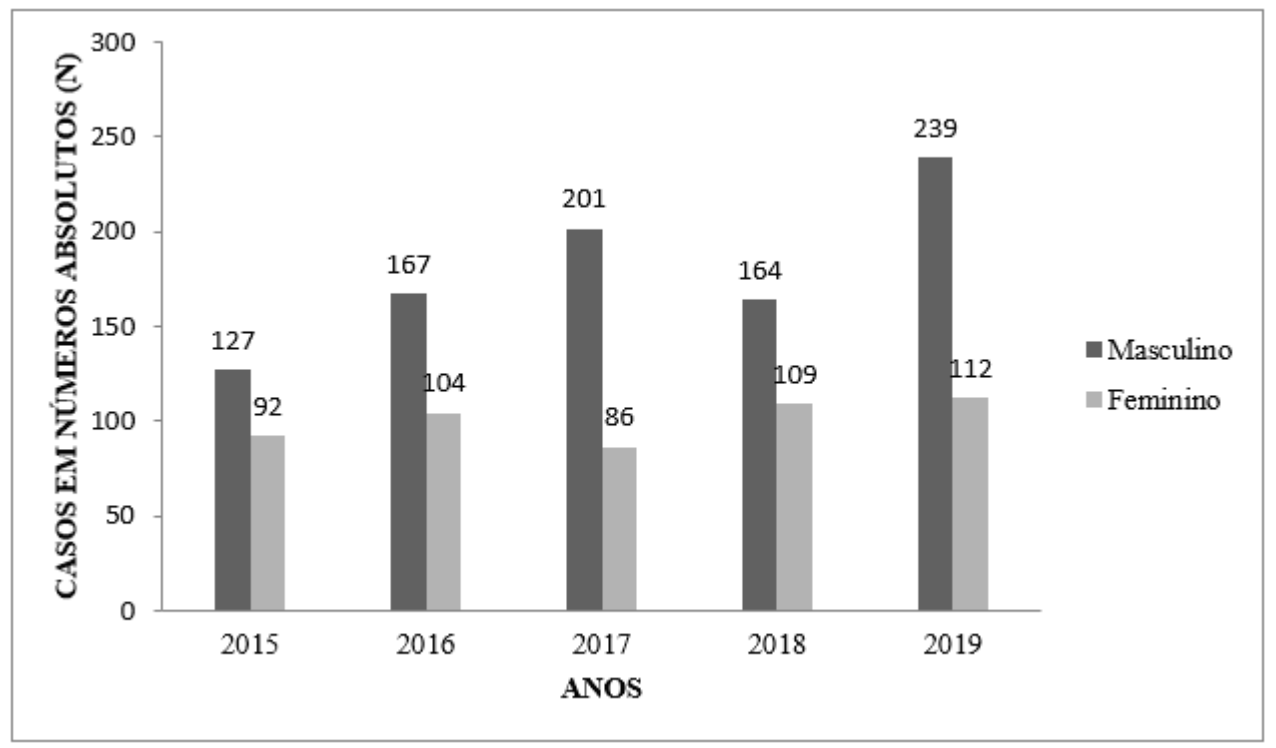

Fonte: Produzido pelos autores de acordo com os dados disponíveis no TABNET, 2020. 
No Gráfico 1, observa-se que no Amapá, os homens foram maioria entre os diagnosticados com tuberculose nesse período, sendo responsável por $64,09 \%$ ( $n=$ 898) dos casos. As mulheres representam $35,91 \%(n=503)$, o que expressa uma proporção aproximada de dois homens infectados para cada mulher.

Entre os anos, 2019 se destaca com a maior porcentagem de infectados, 25,05\% (n = 351), sendo o ano com maior número de casos notificações tanto para os homens quanto para as mulheres.

Gráfico 2- Quantidade de casos confirmados com auxílio do exame laboratorial de acordo com o sexo, entre 2015 e 2019 no Amapá.

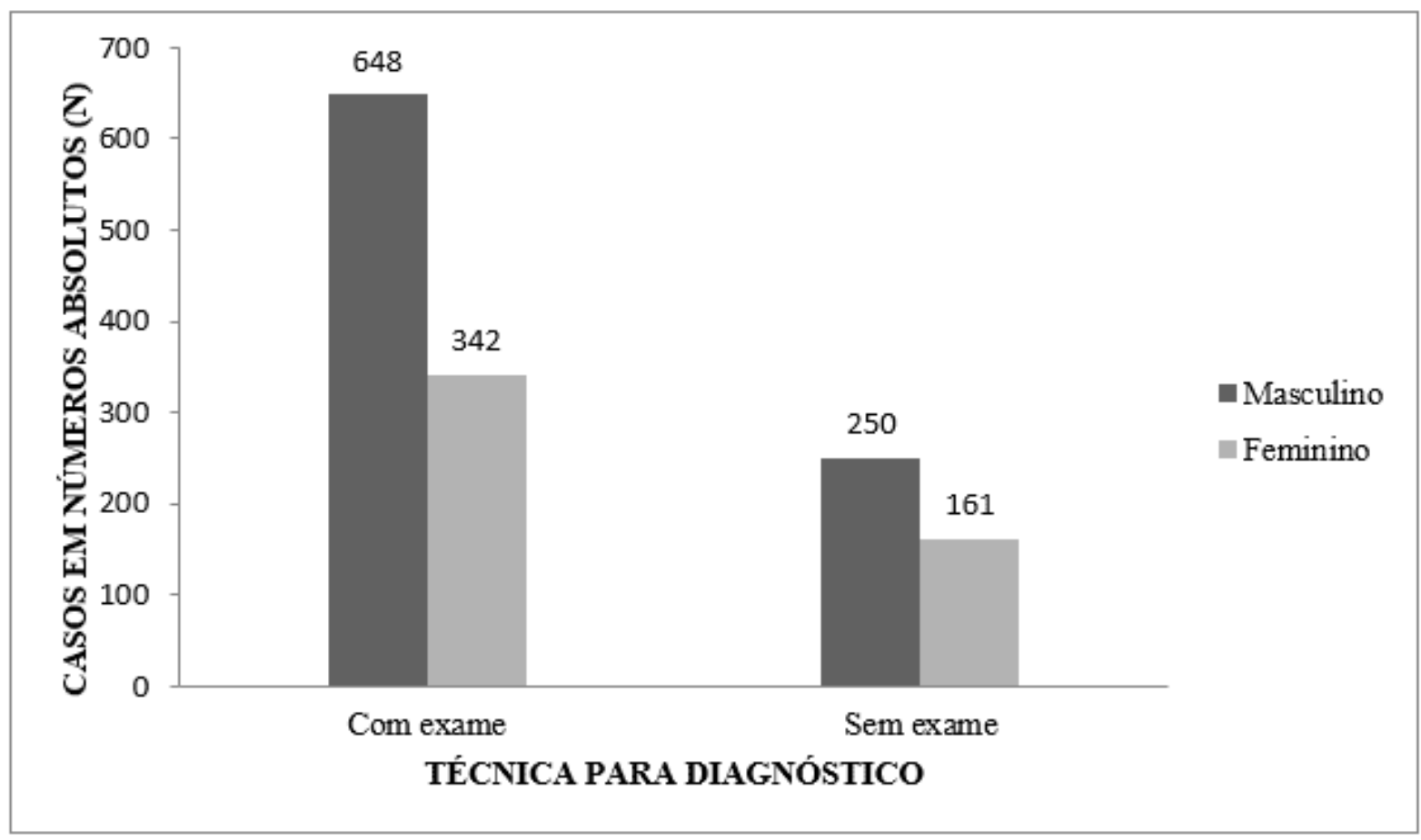

Fonte: Produzido pelos autores de acordo com os dados disponíveis no TABNET, 2020.

No Gráfico 2, é notório que cerca de $70,06 \%(\mathrm{n}=990)$ desses resultados foram baseados no exame laboratorial. Enquanto 29,34\% $(n=411)$ desses diagnósticos foram feitos com base na análise clínica-epidemiológica do paciente. 
Quando se compara a situação dos pacientes separadamente pelo sexo, nos homens $72,16 \%$ ( $n=648$ ) obtiveram confirmação da infecção por meio do exame laboratorial. Já entre as mulheres, $67,99 \%(n=342)$ confirmaram a presença da infecção pela análise laboratorial.

Gráfico 3 - Quantidade de casos confirmados pela primeira baciloscopia do escarro de acordo com o sexo, entre 2015 e 2019 no Amapá.

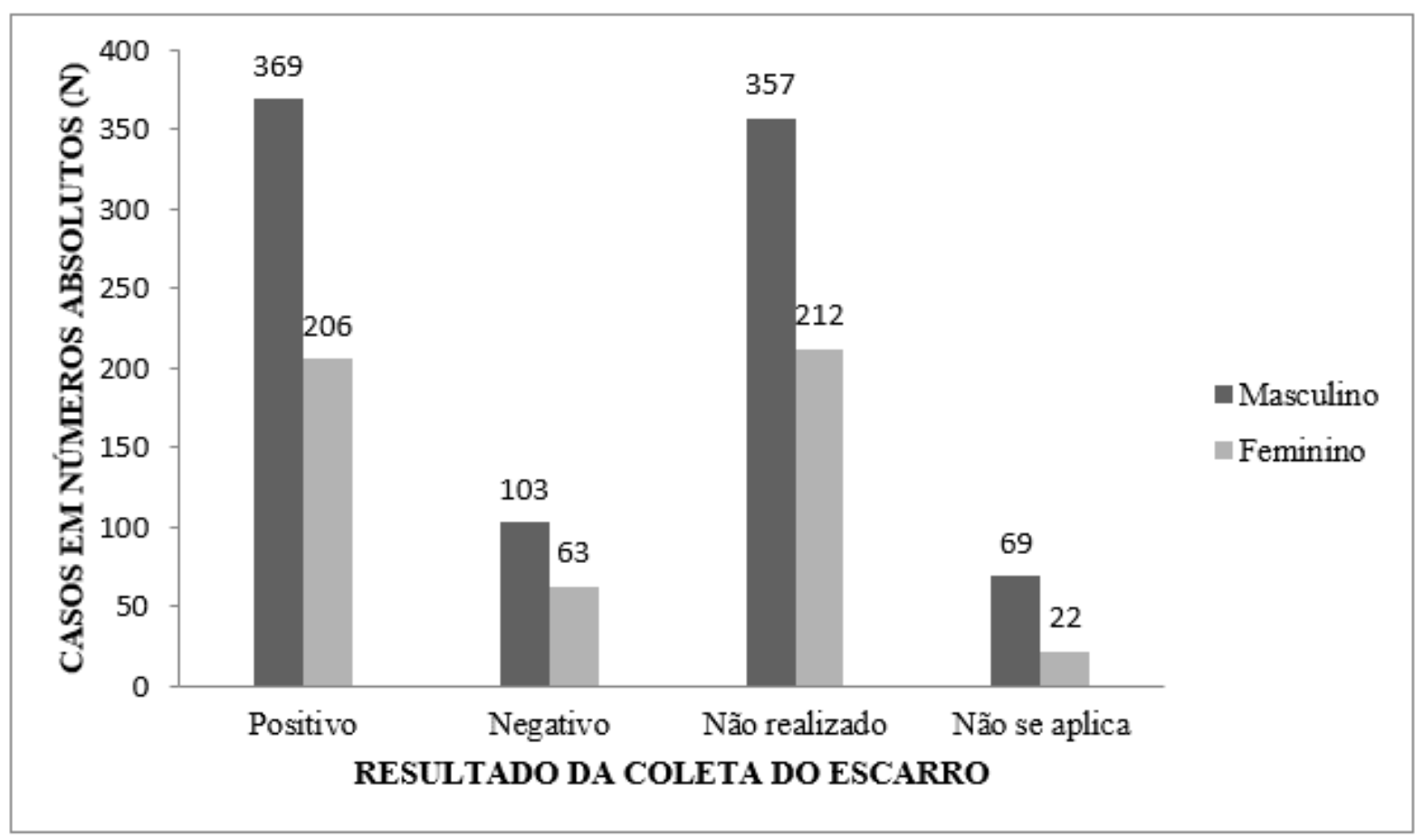

Fonte: Produzido pelos autores de acordo com os dados disponíveis no TABNET, 2020.

O Gráfico 3 ilustra a coleta de escarro para auxiliar do diagnóstico de tuberculose no sexo masculino e feminino. Diante disso, é perceptível que entre os homens, cerca de $52,50 \%(n=472)$ realizaram a coleta do escarro. Desta coleta, $78,17 \%(n=369)$ testaram positivo e $21,83 \%(n=103)$ negativo. Além disso, 39,97\% $(n=357)$ não realizaram a coleta do escarro.

Em contrapartida, 53,47\% $(n=269)$ das mulheres diagnosticadas no Amapá realizaram a primeira baciloscopia por escarro. Entre as mulheres que realizaram a 
coleta, $76,57 \%(n=206)$ testaram positivo e $23,43 \%(n=63)$ testaram negativo. Ademais, nota-se que em $42,14 \%$ ( $n=212)$ não houve a coleta do escarro.

Gráfico 4 - Quantidade de casos confirmados pela segunda baciloscopia do escarro de acordo com o sexo, entre 2015 e 2019 no Amapá.

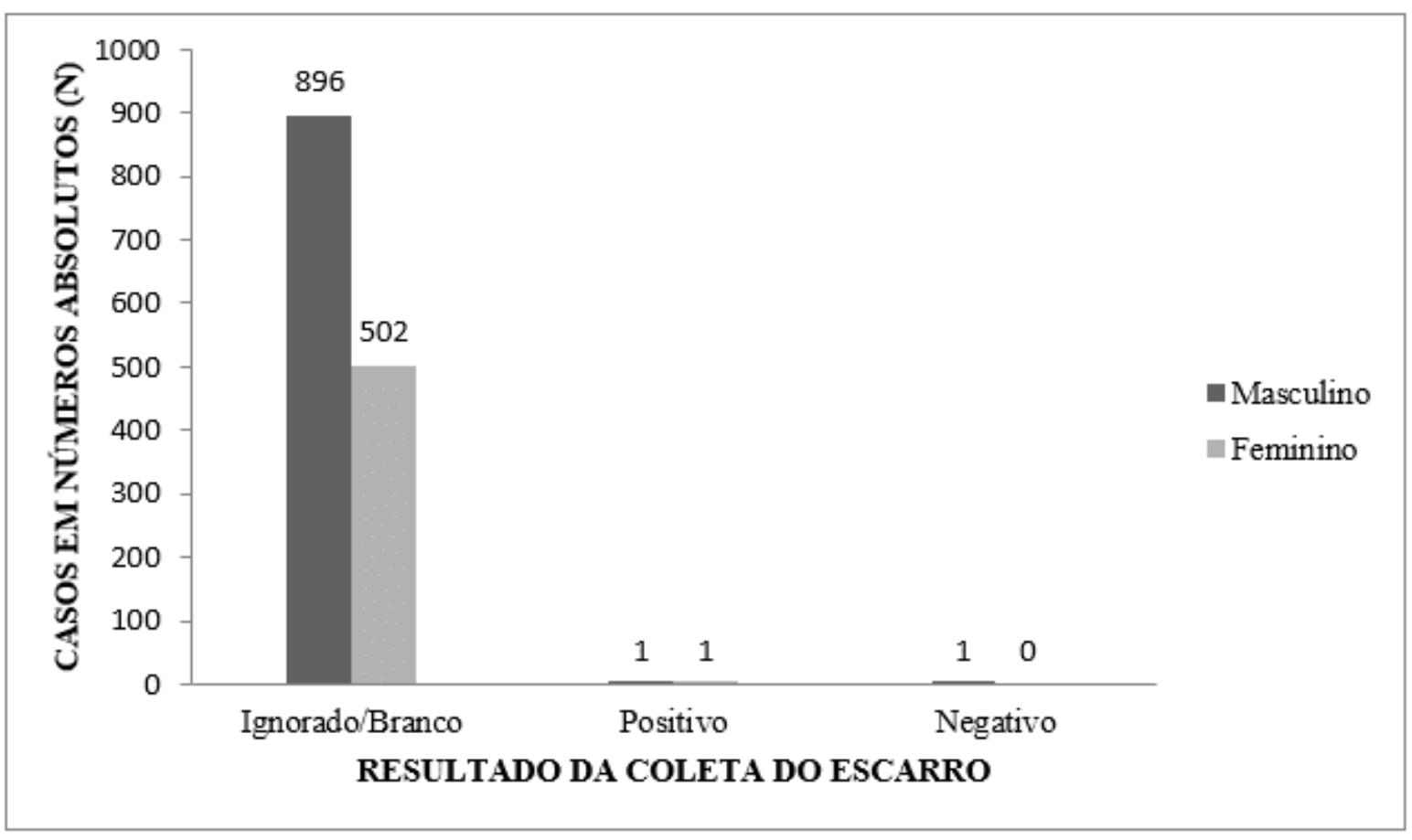

Fonte: Produzido pelos autores de acordo com os dados disponíveis no TABNET, 2020.

O Gráfico 4 revela que no Amapá 99,36\% $(n=1398)$ dos casos confirmados de tuberculose não realizaram a segunda baciloscopia do escarro para diagnóstico.

Essa situação é similar tanto no sexo masculino, no qual apenas $0,22 \%(n=2)$ realizaram a baciloscopia. Já no sexo feminino, apenas $0,19 \%(n=1)$ realizaram o exame. 
Gráfico 5 - Quantidade de casos controle por baciloscopia no $2^{\circ}$ mês em tratamento de acordo com o sexo, entre 2015 e 2019 no Amapá.

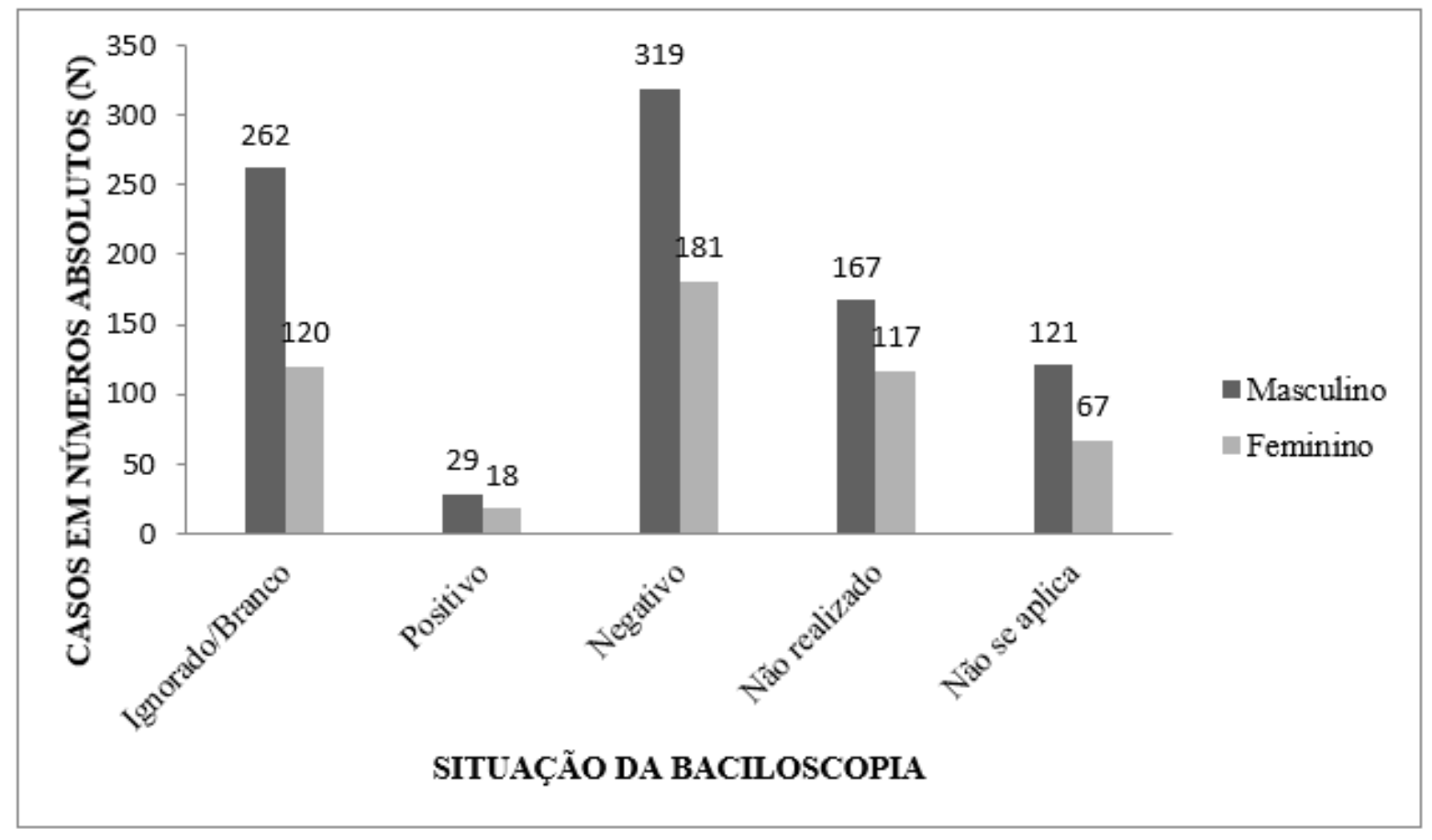

Fonte: Produzido pelos autores de acordo com os dados disponíveis no TABNET, 2020.

O Gráfico 5 aponta que em 39,04\% ( $n=547)$ dos casos confirmados foram realizados a baciloscopia. Entre esses pacientes que realizaram o teste, fazendo o recorte apenas do sexo feminino, mostrou-se negativo em $90,95 \%(n=181)$ dos exames. Enquanto dos casos exclusivamente masculino, mostrou-se negativo em $91,66 \%$ ( $n=$ 319) dos exames.

Além disso, houve uma quantidade expressiva de casos nos quais o acompanhamento pela baciloscopia foi ignorado ou não realizado, o que representou $47,53 \%(n=666)$ das notificações encontradas no SINAN. 
Gráfico 6 - Quantidade de casos controle por baciloscopia no 60 mês em tratamento de acordo com o sexo, entre 2015 e 2019, no Amapá.

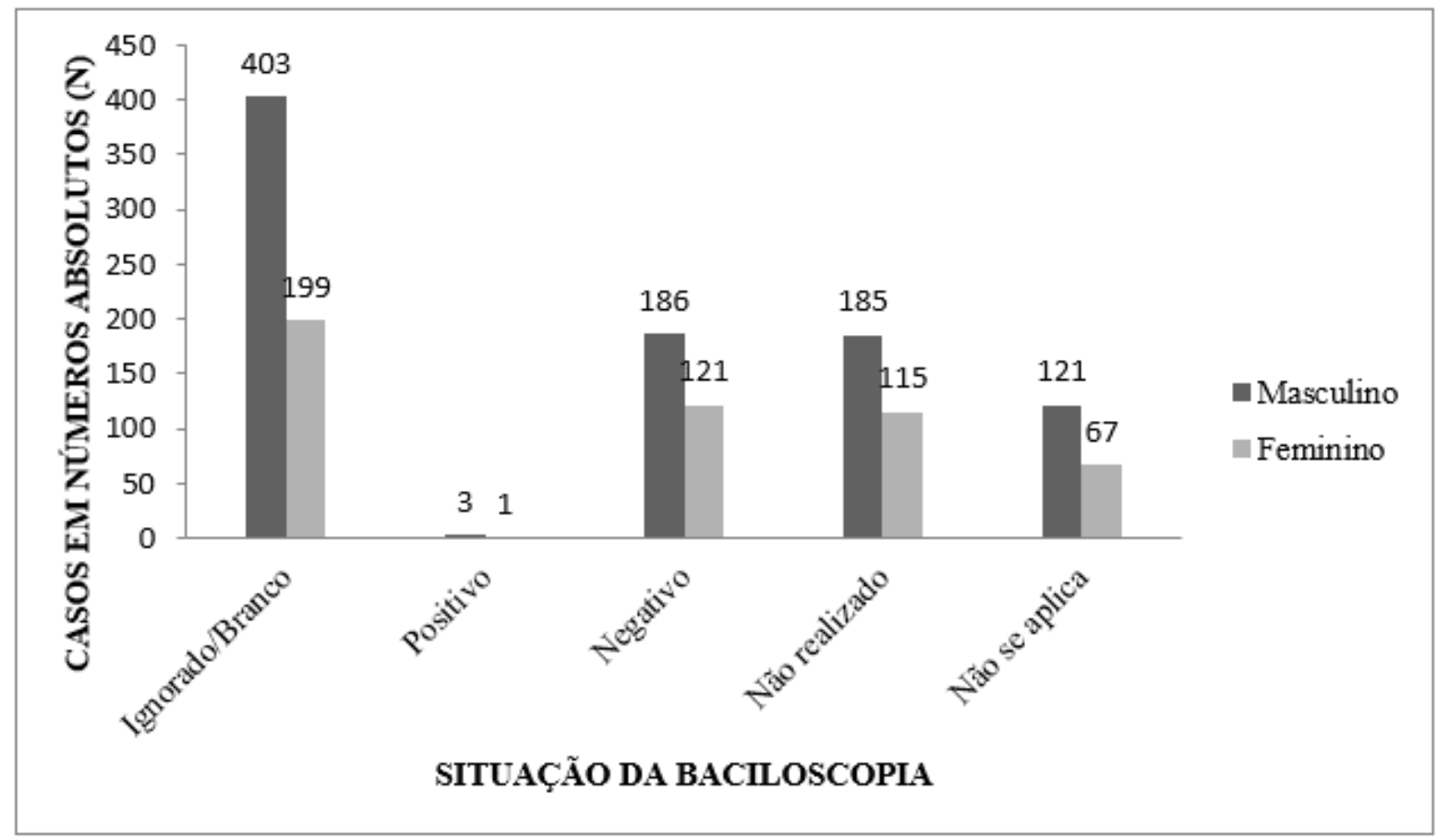

Fonte: Produzido pelos autores de acordo com os dados disponíveis no TABNET, 2020.

No Gráfico 6 observa-se que em apenas 21,19\% $(n=311)$ dos casos confirmados foi realizada a baciloscopia no fim do sexto mês em tratamento.

Entre esses pacientes que fizeram o teste no fim do sexto mês, no sexo masculino mostrou-se negativo em $98,41 \%(n=186)$ dos exames, enquanto no feminino mostrou-se negativo em $99,18 \%(n=115)$ dos exames.

Os casos ignorados e não realizados, representam a maioria das notificações, sendo responsável por $64,38 \%(n=902)$ destas. 
Gráfico 7 - Quantidade de casos confirmados por situação encerrada de acordo com o sexo, entre 2015 e 2019 no Amapá.

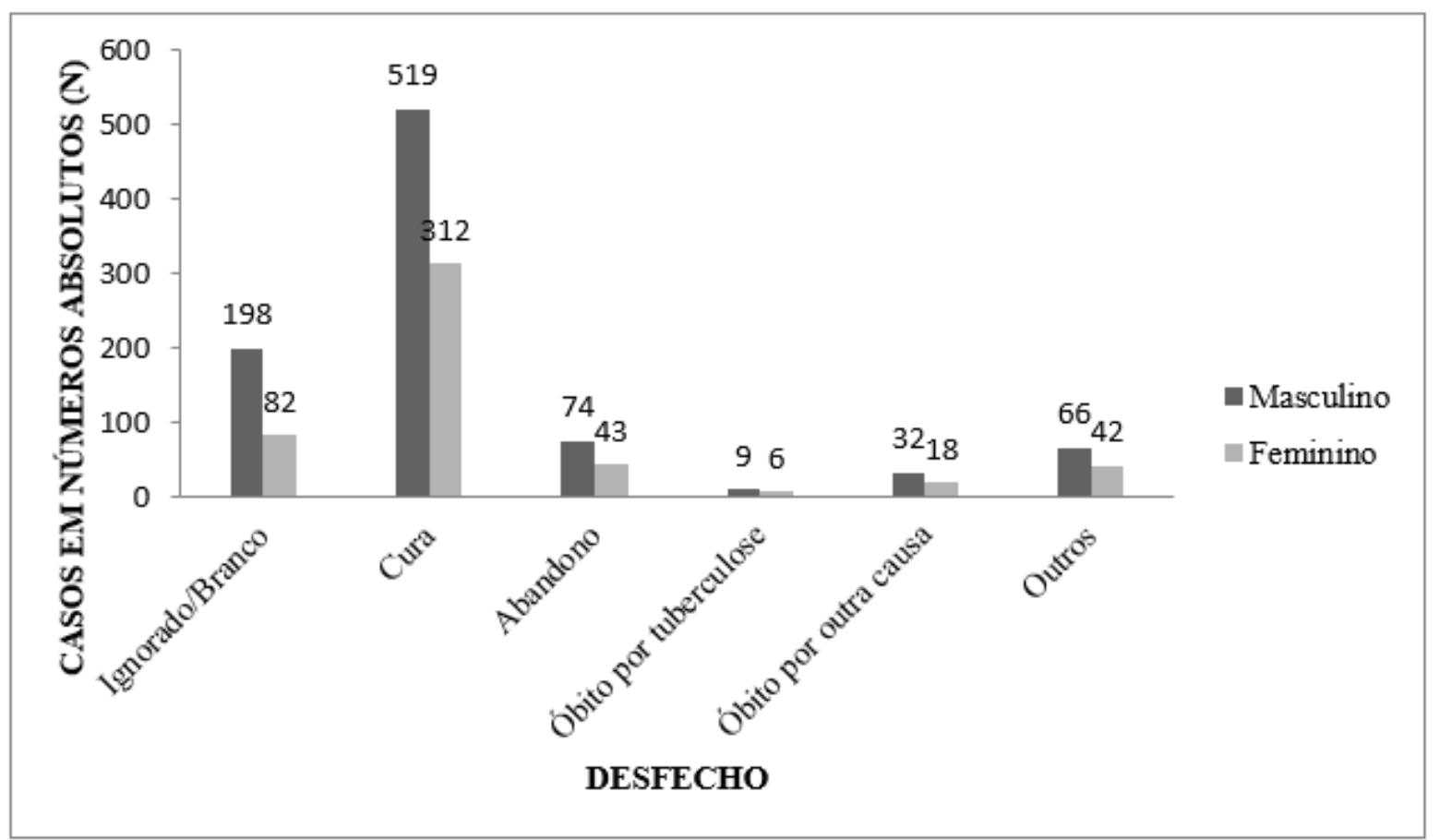

Fonte: Produzido pelos autores de acordo com os dados disponíveis no TABNET, 2020.

No Gráfico 7 observa-se que entre a população masculina houve 57,79\% ( $n=519)$ de cura; $8,20 \%(n=74)$ de abandono; 3,56\% ( $n=32)$ óbitos por outra causa e 1,00\% ( $n$ =9) de óbitos por tuberculose.

Em contrapartida, na população feminina houve $62,02 \%(n=312)$ de cura; 8,54\% ( $n$ $=43)$ de abandono; $3,57 \%(n=18)$ de óbitos por outras causas e $1,19 \%(n=6)$ de óbitos por tuberculose. 
Gráfico 8 - Quantidade de casos confirmados por faixa etária segundo situação encerrada, entre 2015 e 2019 no Amapá.

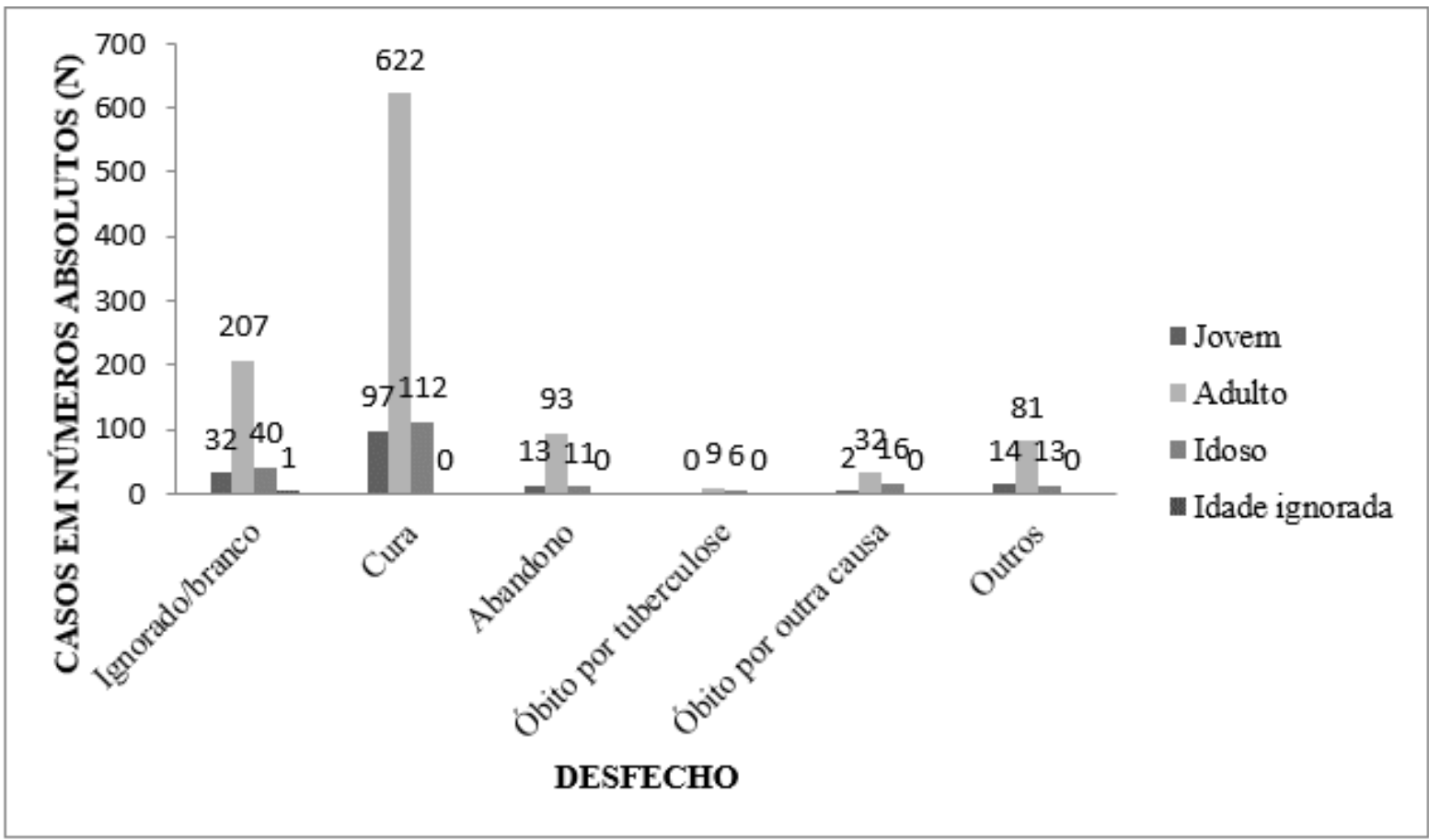

Fonte: Produzido pelos autores de acordo com os dados disponíveis no TABNET, 2020.

O "Gráfico 8" expressa que entre a população jovem (0 a 19 anos) que representa $11,27 \%(n=158)$ dos casos, houve $61,39 \%(n=97)$ de cura, sendo a grupo com a maior porcentagem de curados; $8,22 \%$ de abandono $(n=13) ; 1,26 \%(n=2)$ de óbitos por outra causa e $0,00 \%(n=0)$ de óbitos por tuberculose.

Já entre os adultos (20 a 59 anos) que representam 74,51\% $(n=1044)$ dos casos, houve $59,57 \%(n=622)$ de cura; $8,90 \%(n=93)$ de abandono, sendo o grupo que apresenta a maior taxa de abandono; $3.06 \%(n=32)$ de óbitos por outra causa e $0,86 \%(n=9)$ de óbitos por tuberculose.

Entre os idosos (maiores de 60 anos) que representam 14,14\% $(n=198)$ dos casos, houve $56,56 \%(n=112)$ de cura; $5,55 \%(n=11)$ de abandono; $8,08 \%(n=16)$ de óbitos por outra causa e $3,03 \%(n=6)$ de óbitos por tuberculose, de forma que 
representa o grupo com maior índice de mortalidade pela tuberculose, apesar de apresentar a menor taxa de abandono do tratamento.

\section{DISCUSSÃO}

Ao analisar os dados sobre a tuberculose no Estado do Amapá, percebe-se que os números (Gráfico 1) acompanham a tendência nacional do aumento gradativo, de forma geral, na quantidade de casos no período recortado para o estudo, em que o sexo masculino apresenta o maior número de diagnósticos, resultando-se em uma margem de 1,5 a 2 casos entre homens para cada mulher (BRASIL, 2019). Os números superiores encontrados na população masculina podem ser atribuídos a questões relacionadas ao sexo, uma vez que essa população se encontra mais vulnerável à doença por apresentarem em maior grau fatores de risco, como alcoolismo, coinfecção com o vírus da imunodeficiência humana (HIV), tabagismo e uso de drogas em geral (LEAL; MACIEL; CADE, 2019).

A situação dos diagnósticos da tuberculose no Estado do Amapá se mostra deficitária. Observa-se que aproximadamente dois terços dos pacientes (Gráfico 2), de ambos os sexos, obtiveram o diagnóstico laboratorial, como é proposto pelo Ministério da Saúde (MS) para comprovar a presença do bacilo de Koch no organismo, inferindo-se uma quantidade significativa de diagnósticos clinico e epidemiológicos, realizados quando o quadro clínico é típico e o contexto epidemiológico é apropriado. Desse modo, se demonstra notificações tardias da doença (BRASIL, 2019).

Para a realização de um diagnóstico precoce da tuberculose, o papel do agente comunitário de saúde (ACS) é de suma importância, uma vez que esses profissionais podem observar os primeiros sintomas nas visitas domiciliares, assim, sensibilizar a população da necessidade de uma consulta médica e fazer solicitação de exames como a baciloscopia de escarro para a confirmação da enfermidade. Para que isso ocorra, o ACS deve estar capacitado com um grau de conhecimento satisfatório acerca da tuberculose, possibilitando mais agilidade nas notificações (GASPAR et al., 2019). 
Todavia, esse exame se mostra com pouca adesão no Amapá, principalmente no sexo masculino, sendo o primeiro escarro presente apenas em cerca de metade dos diagnósticos (Gráfico 3) e praticamente nula a confirmação feita pela segunda amostra do escarro (Gráfico 4), o que pode ser interpretado como lacunas na atenção primária à saúde do estado do Amapá, as quais podem estar presentes tanto no processo de investigação da doença, como falhas na comunicação entre a equipe multiprofissional e o usuário da UBS acerca das datas para o exame, quanto na negligência da solicitação da baciloscopia (CARDOSO, 2020).

O acompanhamento dos casos confirmados no Amapá também se mostra deficiente. Pouco mais de um terço dos pacientes realizaram a baciloscopia após dois meses de tratamento (Gráfico 5) e cerca de uma a cada cinco pessoas diagnosticadas fizeram a baciloscopia após seis meses de tratamento (Gráfico 6). Desses pacientes que realizaram o tratamento, obtiveram-se resultados satisfatórios, aproximadamente nove curas para cada dez, tanto do sexo masculino quanto do sexo feminino, no estado do Amapá. Todavia, o processo de adesão ao tratamento é um dos principais desafios encontrado pelas equipes de saúde no combate à tuberculose, já que demanda um grande período fazendo o uso das medicações e, socialmente, há estigmas sobre esse grupo, contribuindo para abandono do tratamento, de modo que possa selecionar bactérias resistentes aos medicamentos e assim um pior prognóstico da doença (FERNANDES et al., 2020). Diante disso, a atenção primária à saúde amapaense apresenta-se como uma aliada na estratégia de aumento ao número de pacientes que irão aderir ao tratamento, tendo em vista que essa tarefa é multifatorial, indo além da distribuição dos medicamentos, a equipe multiprofissional tem o papel de conscientizar e desmistificar possíveis dúvidas nos pacientes menos esclarecidos sobre a doença (LINHARES; PAZ, 2020).

A situação encerrada dos casos de tuberculose no Amapá mostra-se com piores resultados de cura para a população masculina, uma vez que apenas um pouco mais da metade dos homens infectados apresentaram esse desfecho, enquanto as mulheres que se curaram apresentaram proporções próximas de dois terços (Gráfico 7). Apesar do maior índice de mulheres curadas, as proporções, entre ambos os 
gêneros, de abandono e morte pela tuberculose ou por outra causa são similares no estado do Amapá, favorecendo a hipótese que mesmo aderindo ao tratamento por seis meses (Gráfico 5, Gráfico 6), os fatores de risco, presentes com maior frequência na população masculina, diminuem a probabilidade de cura do paciente com tuberculose. No entanto, negligenciar a medicação, independente do sexo, apresenta um pior prognóstico, até mesmo na população que não se expõem aos fatores de risco (ABREU et al., 2020).

Em relação à faixa etária dos casos amapaenses com situação encerrada, as proporções de cura diminuíram progressivamente conforme a idade dos pacientes aumentou (Gráfico 8). A população jovem do Amapá representou os menores índices de casos confirmados, com as maiores porcentagens de cura, com uma proporção maior do que seis para cada dez infectados. Não foi registrada nenhuma morte pela tuberculose, essa realidade pode ser atribuída a características do sistema imunológico desse grupo que, de forma geral e sem comorbidade, apresenta uma maior resistência à infecção (SOUSA et al., 2019).

A população adulta (Gráfico 8) representou o maior índice de infectados, próximo de três para cada quatro casos, e a proporção de cura próxima de seis para cada dez infectados, e foi a população com maior índice de abandono do tratamento, apresentando o índice de morte pela tuberculose próximo de nove para cada cem infectados. Entre os fatores que contribuem para esses números, a maior atividade laboral dessa classe contribui para maior exposição a aglomerações, facilitando a propagação da bactéria (JESUS et al., 2020).

A população idosa (Gráfico 8) apresentou os menores índices de cura, sendo cerca de um pouco mais da metade dos infectados e os menores índices de abandono do tratamento, todavia, foi a população com a maior porcentagem de mortes pela infecção, sendo acima do triplo da população adulta. Entre os fatores relacionados à senescência que podem justificar essas estatísticas estão a capacidade reduzida de regeneração e produção celular, o que contribui para maior vulnerabilidade sistêmica do organismo e a presença de comorbidade crônicas como o diabetes de mellitus (RODRIGUES; TAUIL, 2019). 
A presença do grande número de "Ignorados/branco" foi considerada um limitador do estudo, uma vez que dificultou uma análise mais precisa dos dados fornecidos pelo TABNET sobre a tuberculose no estado do Amapá, mostrando que a notificação amapaense apresenta falhas recorrentes no preenchimento das variáveis propostas pelo SINAN, corroborando para resultados com uma menor precisão. Apesar disso, os demais dados analisados se mostram suficientes para que o perfil amapaense da adesão ao tratamento da tuberculose seja traçado, contribuindo de forma efetiva com a comunidade científica (ROCHA et al., 2020).

\section{CONSIDERAÇÕES FINAIS}

O perfil epidemiológico é uma ferramenta de estudo populacional extremamente eficaz no que tange ao conhecimento em saúde de uma população, analisando suas particularidades e subjetividades, a partir do qual se pode ampliar estratégias à assistência.

Este estudo realizou análise de dados acerca da adesão ao tratamento de tuberculose no estado do Amapá entre os anos de 2015 e 2019, e infere-se que os dados disponíveis demonstram padrão esperado de aumento no número de casos, seguindo a tendência nacional, além de que os homens são mais acometidos do que as mulheres. Ademais, a realidade acerca da adesão ao tratamento, o qual é inteiramente disponibilizado pelo ministério da saúde, é preocupante, pois os desfechos esperados não superam a expectativa de cura, pelo contrário, demonstram diminuição da adesão ao longo do acompanhamento ou a falta de dados.

Importante observar que, conforme a literatura, pacientes com comorbidades ou fatores de risco prévios possuem um desfecho pior, como as pessoas idosas. Junto a isso, é possível observar pelo estudo que existe uma forte tendência no estado de subnotificação ao longo da doença, ou mesmo durante o diagnóstico, o que prejudica a quebra da cadeia de transmissão e o pior desfecho de pacientes diagnosticado tardiamente. 
Diante disso, é importante que as autoridades em saúde das esferas municipais, estadual e federal atentem aos dados progressivos que a população apresenta e tome medidas mais eficazes como a busca ativa por sintomáticos respiratórios, pessoas em situação de rural ou privadas de liberdade, profissionais de saúde, imunossuprimidos e idosos, principalmente os expostos na comunidade por meio de rastreamento por radiografia de tórax e realização de prova tuberculínica nos grupos supracitados. Deve-se frisar que o sistema único de saúde brasileiro possui planejamento de medidas de prevenção, detecção e terapêutica muito eficazes e um alinhamento de planejamento entre gestores é necessário ao combate da doença no país.

\section{REFERÊNCIAS}

ABREU, Ricardo Gadelha de et al . Tuberculose e diabetes: associação com características sociodemográficas e de diagnóstico e tratamento. Brasil, 20072011. Rev. bras. epidemiol., Rio de Janeiro, v. 23, e200009, 2020.

BRASIL. Secretaria de Vigilância em Saúde. MINISTÉRIO DA SAÚDE. Boletim Epidemiológico. [S. I.], 2020.

. MINISTÉRIO DA SAÚDE. Secretaria de Vigilância em Saúde. Departamento de Vigilância Epidemiológica. Manual de recomendações para o controle da tuberculose no Brasil. 2. ed. atual. BRASILIA: [s. n.], 2019. 364 p.

. MINISTÉRIO DA SAÚDE. Sistema de informação de agravos e notificação. Tuberculose - Notificações Registradas: banco de dados. Disponível em: http://tabnet.datasus.gov.br/cgi/tabcgi.exe?sinannet/cnv/tubercap.def. Acesso em: 14 jul. 2020.

. MINISTÉRIO DA SAÚDE. Tuberculose: os desafios do tratamento contínuo. [S. I.], 2019.

. MINISTÉRIO DA SAÚDE. Secretaria de Vigilância em Saúde Departamento de Vigilância Epidemiológica. Protocolo de vigilância da infecção latente pelo Mycobacterium tuberculosis no Brasil. [S. I.], 2018. 
MINISTÉRIO DA SAÚDE. Secretaria de Vigilância em Saúde Departamento de Vigilância Epidemiológica. Cartilha para o Agente Comunitário de Saúde: Tuberculose. [S. I.], 2017.

CARDOSO, Gisela Cordeiro Pereira et al . Sítios simbólicos de pertencimento e prevenção e controle da tuberculose: percepções e práticas dos Agentes Comunitários de Saúde no Brasil e na Etiópia. Ciênc. saúde coletiva, Rio de Janeiro , v. 25, n. 8, p. 2927-2937, Ago. 2020.

CARVALHO, A. C. C. et al. Aspectos epidemiológicos, manifestações clínicas e prevenção da tuberculose pediátrica sob a perspectiva da estratégia End TBv. J Bras Pneumol., v. 44, n. 2, p. 134-144, 2018.

DA SILVA, M. E. N.; DE LIMA, D. S.; DOS SANTOS, J. E.; MONTEIRO, A. C. F.; TORQUATO, C. M. M.; FREIRE, V. A.; RIBEIRO, D. B. C.; FEITOSA, A. C. S.; TEIXEIRA, A. B. Aspectos gerais da tuberculose: uma atualização sobre o agente etiológico e o tratamento. Revista Brasileira de Análises Clínicas, [s. I.], 2018.

FERNANDES, Thauana dos Santos et al . Estigma e preconceito na atualidade: vivência dos portadores de tuberculose em oficinas de terapia ocupacional. Physis, Rio de Janeiro, v. 30, n. 1, e300103, 2020.

FOCACCIA, Roberto. Tratado de Infectologia. 5. ed. rev. e atual. [S. I.]: Atheneu, 2015.

GASPAR, Lucijane Maria da Silva et al . Conhecimento, atitudes e práticas de agentes comunitários de saúde sobre tuberculose pulmonar em uma capital do Nordeste do Brasil. Ciênc. saúde coletiva, Rio de Janeiro, v. 24, n. 10, p. 3815-3824, Out. 2019

JESUS, Michele da Cruz de. Et al. Casos confirmados de tuberculose no Brasil, na Região Norte, no Estado do Amapá e no Município de Macapá, entre 2013 e 2017. Revista Científica Multidisciplinar Núcleo do Conhecimento. Ano 05 , Ed. 05 , Vol. 01, pp. 144-154. Maio de 2020. ISSN: 2448-0959, 
KOZAKEVICH, G. V.; SILVA, R. M. D. Tuberculose: Revisão de Literatura. Arq. Catarin Med. , v. 44, n. 4, p. 34-47, 2015.

LEAL, Marcelle Lemos; MACIEL, Ethel Leonor Nóia; CADE, Nágela Valadão. Fatores associados à tuberculose em população de diabéticos: um estudo casocontrole. Ciênc. saúde coletiva, Rio de Janeiro , v. 24, n. 9, p. 32473256, Set. 2019 .

LINHARES, Shirley Ribeiro dos Santos; PAZ, Elisabete Pimenta Araújo. A vivência do tratamento de tuberculose em unidades de Saúde da Família. Esc. Anna Nery, Rio de Janeiro, v. 24, n. 2, e20190209, 2020.

NOGUEIRA, Péricles Alves; ABRAHÃO, Regina Maura Cabral de Melo; GALESI, Vera Maria Neder; LÓPEZ, Rossana Verónica Mendoza. Tuberculose e infecção latente em funcionários de diferentes tipos de unidades prisionais. Rev Saude Publica. 2017 ;51:13.

ROCHA, Marli Souza et al . Sistema de Informação de Agravos de Notificação (Sinan): principais características da notificação e da análise de dados relacionada à tuberculose. Epidemiol. Serv. Saúde, Brasília, v. 29, n. 1, e2019017, 2020.

RODRIGUES, Olga Maíra Machado; TAUIL, Pedro Luiz. Aspectos clínicos e epidemiológicos da tuberculose no Distrito Federal (2006 a 2015). Rev. bras. epidemiol., São Paulo, v. 22, e190055, 2019.

SOUSA, George Jó Bezerra et al . Clinical and epidemiological features of tuberculosis in children and adolescents. Rev. Bras. Enferm., Brasília , v. 72, n. 5, p. 12711278, Out. 2019 .

Enviado: Setembro, 2020.

Aprovado: Outubro, 2020. 